\title{
The role of extended-spectrum cephalosporin-resistance in recurrent community-onset Enterobacteriaceae urinary tract infections: a retrospective cohort study
}

Judith A. Anesi ${ }^{1 *}$ D, Ebbing Lautenbach², Irving Nachamkin³, Charles Garrigan³, Warren B. Bilker ${ }^{4}$, Jacqueline Omorogbe ${ }^{5}$, Lois Dankwa ${ }^{5}$, Mary Wheeler ${ }^{5}$, Pam Tolomeo ${ }^{5}$, Jennifer H. Han ${ }^{2}$ and for the CDC Prevention Epicenters Program

\begin{abstract}
Background: Bacterial resistance to first line antibiotics used to treat community-onset urinary tract infections (UTIs) continues to emerge. We sought to determine the association between extended-spectrum cephalosporin resistance (ESC-R) and recurrence among Enterobacteriaceae (EB) UTIs.

Methods: A retrospective cohort study was performed. All patients presenting to the Emergency Departments (EDs) or outpatient practices in a large health system with EB UTIs between 2010 and 2013 were included. Exposed patients had ESC-R EB UTIs. Unexposed patients had ESC-susceptible EB UTIs and were matched to exposed patients 1:1 on study year. Multivariable Cox proportional hazards regression analyses were performed to evaluate the association between ESC-R EB UTI and time to recurrent UTI within 12 months.

Results: A total of 302 patients with an index community-onset EB UTI were included, with 151 exposed and 151 unexposed. Overall, 163 (54\%) patients experienced a recurrent UTI with a median time to recurrence of 69 days (interquartile range 25-183). On multivariable analyses, ESC-resistance was associated with an increased hazard of recurrent UTI (hazard ratio [HR] 1.39, 95\% confidence interval [CI] 1.01-1.91, $P=0.04$ ). Other variables that were independently associated with recurrence included a history of UTI prior to the index UTI and presence of a urinary catheter at the time of the index UTI. Secondarily, we found that when the treatment for the index UTI was adjusted for, there was no longer a significant association between ESC-R status and time to recurrent UTI (aHR 1. $26,95 \% \mathrm{Cl} 0.91-1.76, P=0.17)$.
\end{abstract}

Conclusions: Community-onset UTI due to EB demonstrating ESC-resistance is associated with a significantly increased hazard of recurrent UTI within 12 months compared to ESC-susceptible EB, even after adjusting for baseline factors that predispose patients to UTI recurrence. This association appears to be driven primarily by delayed or inappropriate treatment for the index ESC-R EB UTI.

Keywords: Community-onset, Urinary tract infection, Enterobacteriaceae, Extended-spectrum beta-lactamase (ESBL), Extended-spectrum cephalosporin-resistant

\footnotetext{
* Correspondence: judith.anesi@uphs.upenn.edu

'Division of Infectious Diseases, Department of Medicine; Center for Clinical

Epidemiology and Biostatistics, Perelman School of Medicine, University of

Pennsylvania, 719 Blockley Hall, 423 Guardian Drive, Philadelphia, PA 19104,

USA

Full list of author information is available at the end of the article
}

(c) The Author(s). 2019 Open Access This article is distributed under the terms of the Creative Commons Attribution 4.0 International License (http://creativecommons.org/licenses/by/4.0/), which permits unrestricted use, distribution, and reproduction in any medium, provided you give appropriate credit to the original author(s) and the source, provide a link to the Creative Commons license, and indicate if changes were made. The Creative Commons Public Domain Dedication waiver (http://creativecommons.org/publicdomain/zero/1.0/) applies to the data made available in this article, unless otherwise stated. 


\section{Background}

Urinary tract infections (UTIs) are the most common bacterial infection among adults in the community [1], and there is significant bacterial resistance to first-line antibiotics used to treat UTIs in ambulatory settings [2]. In particular, there have been increasing reports of extended-spectrum cephalosporin-resistant (ESC-R) Enterobacteriaceae (EB) UTIs in the outpatient setting [3-7].

Recurrence is a frequent complication of UTI, with the risk for recurrence varying by population. In post-menopausal women, the risk for recurrence is as high as $80 \%$ in the following 12 months, with a mean of three recurrences during this period [8]. The risk is lower in men but remains notable with one study showing that the risk of recurrence within 12 months is $8 \%$ [9]. Known risk factors for recurrent UTI include female gender, sexual activity, diabetes mellitus, obesity, and anatomic abnormalities of the genitourinary tract [10].

Relatively little is known about the role that multidrug-resistant organisms (MDROs), and specifically ESC-resistance, play in recurrent UTIs. Prior studies have shown that patients with recurrent UTIs are more likely to ultimately develop an infection due to an MDRO, including ESC-R EB, likely related to repeated antibiotic exposures [5, 11]. However, it is unknown whether an infection due to an MDRO is in itself more likely to lead to recurrent UTI. To our knowledge, there are no prior studies that have evaluated whether an index UTI due to an ESC-R or extended-spectrum beta-lactamase (ESBL)-producing EB organism is associated with increased risk for UTI recurrence. Therefore, in this study, we sought to determine the association between community-onset ESC-R EB UTI and time to recurrent UTI.

\section{Methods}

\section{Study design and setting}

A retrospective cohort study was performed at two Emergency Departments (EDs) and a network of outpatient practices within the University of Pennsylvania Health System (UPHS), as follows: [1] the ED at the Hospital of the University of Pennsylvania (HUP), a 776-bed quaternary care medical center; [2] the ED at Penn Presbyterian Medical Center (PPMC), a 331-bed academic medical center, and [3] a network of 246 primary care physicians at over 50 community and hospital-based practices.

\section{Study population}

The initial source population was composed of all patients presenting to an ED or outpatient practice who had a urine culture positive for EB between December 21, 2010 and April 22, 2013. Potentially eligible patients were identified through the HUP Clinical Microbiology
Laboratory, which processes all cultures from HUP, PPMC, as well as $>90 \%$ of urine cultures from UPHS outpatient practices. A patient was designated as having a community-onset urine culture if it was obtained in the ED, outpatient practices, or within $72 \mathrm{~h}$ of hospital admission. Subsequently, patients were excluded if they were $<18$ years-old, expired during the follow-up period, were a long-term care facility resident, or had a physician who failed to consent. The remaining subjects were eligible and were approached for consent. Those with an ESC-R EB organism on urine culture were approached first, and then a random selection of those with ESC-S EB organism on urine culture were approached in an equal number as those with ESC-R EB. After consenting, only patients with a true UTI were included as we sought to identify outcomes associated with ESC-R EB UTI rather than urinary colonization. A urine culture was considered indicative of an infection based on Centers for Disease Control and Prevention (CDC)/National Healthcare Safety Network (NHSN) criteria [12], which was determined via medical record review performed by an infectious diseases-trained physician (J.H.H.).

Exposed patients were defined as those with an EB UTI demonstrating resistance to an ESC (i.e., ceftriaxone or cefotaxime minimum inhibitory concentration $[\mathrm{MIC}]>1$ $\mathrm{mg} / \mathrm{L}$ ) according to Clinical and Laboratory Standards Institute (CLSI) criteria [13]. Unexposed patients were those who had a UTI with ESC-susceptible EB during the study period (i.e., ceftriaxone and cefotaxime MICs $\leq 1 \mathrm{mg} / \mathrm{L}$ ). Unexposed patients were randomly selected from among all patients with ESC-susceptible EB UTIs using a computerized random number generator and were matched with exposed patients in a 1:1 ratio based on study year. This initial ESC-R or ESC-susceptible UTI (the exposure of interest) was designated as the "index UTI". Each patient was included as a subject only once. The study was approved by the institutional review board of the University of Pennsylvania.

\section{Outcome}

The primary outcome was time to first recurrent UTI. Modified CDC/NHSN criteria were used to confirm recurrent UTI [12]; our criteria relied on standard symptoms and signs of UTI along with pyuria on urinalysis but did not require a repeat urine culture, since urine cultures are infrequently sent in the outpatient setting. Further, urine cultures are more likely to be sent for patients with a history of ESC-R EB and would thus bias the analysis of recurrence. The outcome was determined via electronic medical record review by an infectious diseases-trained physician (J.A.A.). The electronic medical record system captures all outpatient, ED, and inpatient visits within the health system. The first clinical episode to meet the criteria for UTI was considered the 
first recurrent UTI following the index UTI. The outcome was assessed through 12 months following the index UTI.

\section{Data collection}

Data on exposed and unexposed patients were abstracted from the UPHS electronic medical record system. Information was collected on demographics (e.g., age, gender, race), comorbidities (e.g., diabetes, malignancy, hemodialysis), urologic disorders (e.g., prior UTIs, urinary catheters), recent skilled nursing facility (SNF) or hospital stay, culture location (ED, inpatient, or outpatient practice), and all inpatient and outpatient antibiotic therapy that was prescribed in the 6 months preceding the UTI and in the 14 days following UTI diagnosis.

\section{Susceptibility testing of Enterobacteriaceae isolates}

Susceptibility testing of EB isolates was performed by the HUP Clinical Microbiology Laboratory. All isolates identified from study subjects were tested as part of routine care for susceptibility to antibiotics using the semi-automated Vitek 2 identification and susceptibility system (bioMerieux, Inc., Durham, NC). Updated MIC breakpoints for ceftriaxone and cefotaxime were used without confirmatory ESBL testing according to CLSI guidelines [13].

\section{Statistical analysis}

Exposed and unexposed patients were characterized by potential confounders, such as demographics, comorbidities, and prior antibiotic use. For these paired data, continuous variables were compared using the Wilcoxon signed rank test, and categorical variables were compared using the McNemar test. Bivariable Cox proportional hazard regression was used to examine the relationship between ESC-R EB UTI and time to recurrent UTI; the standard error was adjusted for clustering by matched pair. Patients were censored at the time of a recurrent UTI, death, or end of follow-up. Variables from bivariable analyses with $P$ values $<0.20$ or confounders of the primary association were considered for inclusion in the final multivariable model. Variables were added based on biologic plausibility. Variables were retained in the final model if they were confounders or if they had a $P$ value of $<0.05$ in the multivariable Cox model. A hazard ratio (HR) and $95 \%$ confidence interval (CI) were calculated to evaluate the strength of any association. All analyses were performed using STATA v.14.0 (StataCorp, College Station, Texas).

\section{Secondary analyses}

In secondary exploratory analyses, we evaluated whether the association between ESC-R EB index UTI and time to recurrent UTI was impacted by prompt administration of antibiotics for the index UTI. Specifically, we evaluated the impact of inappropriate initial antibiotic therapy (IIAT). IIAT was binary and defined as failure to receive an antibiotic to which the organism was susceptible within $48 \mathrm{~h}$ of index urine culture collection.

\section{Results \\ Study population}

There were 2009 unique subjects who grew an Enterobacteriaceae species on a urine culture from an outpatient visit, ED visit, or within $72 \mathrm{~h}$ of hospital admission during the study period. After applying exclusion criteria, there were 887 subjects who were eligible. Of these 887 potential subjects, 574 (65\%) consented to participate in the study. Of these, 151 had an ESC-R EB on urine culture that was consistent with true UTI (rather than colonization) and were thus the final "exposed" group. One hundred fifty-one patients with community-onset UTI due to an ESC-susceptible EB were then matched to the exposed patients based on study year and comprised the final "unexposed" group.

Within this cohort of 302 patients, the median age was 56 years (interquartile range [IQR], 37-68), and 62 (21\%) were men. With the index UTI, 217 (72\%) patients presented to an outpatient practice, while $85(28 \%)$ patients presented to the ED. Baseline characteristics of the source cohort are shown in Table 1 stratified by exposure status.

\section{Association of ESC-R EB UTI with time to recurrent UTI}

Within the entire cohort, 163 (54\%) patients developed a recurrent UTI within 12 months of the index UTI. The median time to recurrent UTI was 69 days (IQR 25183). There was documented follow-up within the UPHS system for 286 (95\%) of the patients, through to the time of recurrent UTI, death, or 12 months of follow-up.

Among the 163 recurrent UTIs, only 32 had an accompanying urine culture at the time of the recurrent UTI diagnosis. Among those 32 with urine cultures, 16 were in the ESC-R EB index UTI group, and 16 were in the ESC-S EB index UTI group. All of the recurrent UTIs with an accompanying urine culture, in both groups, were due to the same organism as was identified on the index urine culture. Eleven (34\%) were due to an ESC-R EB organism, and 2 (6\%) were due to a carbapenem-resistant EB organism; all of these multidrug-resistant EB UTI recurrences occurred in subjects who had had an ESC-R EB index UTI.

In the unadjusted analysis, we found that ESC-R EB UTI was associated with an increased hazard of recurrent UTI (hazard ratio [HR] 1.46, 95\% confidence interval $[C I] 1.08-1.98, P$ value $=0.02$ ). The unadjusted Kaplan-Meier survival curve is shown in Fig. 1 (log rank test $P$ value $=0.02$ ). In the final multivariable model 
Table 1 Baseline characteristics of the study cohort stratified by exposure status

\begin{tabular}{|c|c|c|c|}
\hline Variable & ESC-S EB index UTI (unexposed) ${ }^{a}$ & ESC-R EB index UTI (exposed) & $P$ value \\
\hline \multicolumn{4}{|l|}{ Demographics } \\
\hline Age in years (median, IQR) & $49(27-64)$ & $60(46-70)$ & $<0.01$ \\
\hline Female gender & 132 (87\%) & 108 (72\%) & $<0.01$ \\
\hline Nonwhite race & $81(54 \%)$ & $78(52 \%)$ & 0.73 \\
\hline Culture obtained in outpatient clinic & $119(79 \%)$ & $89(59 \%)$ & $<0.01$ \\
\hline Culture obtained in ED & $29(19 \%)$ & $54(36 \%)$ & $<0.01$ \\
\hline Culture obtained within $72 \mathrm{~h}$ of inpatient admission & $3(2 \%)$ & $8(5 \%)$ & 0.23 \\
\hline \multicolumn{4}{|l|}{ Comorbidities/Exposures } \\
\hline UTI in prior 6 months & $57(38 \%)$ & $65(43 \%)$ & 0.37 \\
\hline Urinary catheter & $9(6 \%)$ & $27(18 \%)$ & $<0.01$ \\
\hline Surgery in prior 6 months & $21(14 \%)$ & $36(24 \%)$ & 0.04 \\
\hline Prostate disease (if male) & $6(32 \%)$ & $20(47 \%)$ & $>0.99$ \\
\hline Liver disease $^{\mathrm{b}}$ & $1(1 \%)$ & $8(5 \%)$ & 0.04 \\
\hline Respiratory disease $^{c}$ & $17(11 \%)$ & $29(19 \%)$ & 0.06 \\
\hline Diabetes mellitus & $14(9 \%)$ & $31(21 \%)$ & 0.01 \\
\hline History of hemodialysis & $1(1 \%)$ & $5(3 \%)$ & 0.22 \\
\hline Malignancy & $11(7 \%)$ & $28(19 \%)$ & $<0.01$ \\
\hline Prior renal transplantation & $6(4 \%)$ & $13(8 \%)$ & 0.11 \\
\hline Rehabilitation or SNF stay in prior 6 months & $3(2 \%)$ & $9(6 \%)$ & 0.11 \\
\hline Hospitalization in prior 6 months & $35(23 \%)$ & $66(44 \%)$ & $<0.01$ \\
\hline \multicolumn{4}{|l|}{ Antibiotic exposures $^{\mathrm{d}}$} \\
\hline Any antibiotic & $84(56 \%)$ & $94(62 \%)$ & 0.24 \\
\hline First-generation cephalosporin & $8(5 \%)$ & $18(12 \%)$ & 0.04 \\
\hline Extended-spectrum cephalosporin & $4(3 \%)$ & $19(13 \%)$ & $<0.01$ \\
\hline Extended-spectrum penicillin & $5(3 \%)$ & $12(8 \%)$ & 0.07 \\
\hline Amoxicillin/clavulanate or ampicillin/sulbactam & $5(3 \%)$ & $11(7 \%)$ & 0.11 \\
\hline Piperacillin-tazobactam & $0(0 \%)$ & $1(1 \%)$ & $>0.99$ \\
\hline Fluoroquinolone & $28(19 \%)$ & $41(27 \%)$ & 0.07 \\
\hline Aztreonam & $0(0 \%)$ & $1(1 \%)$ & $>0.99$ \\
\hline Carbapenem & $1(1 \%)$ & $5(3 \%)$ & 0.02 \\
\hline Aminoglycoside & $1(1 \%)$ & $3(2 \%)$ & 0.62 \\
\hline Nitrofurantoin & $26(17 \%)$ & $28(19 \%)$ & 0.78 \\
\hline TMP-SMX & $19(13 \%)$ & $36(24 \%)$ & 0.02 \\
\hline Fosfomycin & $1(1 \%)$ & $2(1 \%)$ & $>0.99$ \\
\hline \multicolumn{4}{|l|}{ Severity of index UTI } \\
\hline Pyelonephritis at diagnosis & $18(12 \%)$ & $44(29 \%)$ & $<0.01$ \\
\hline BSI at diagnosis & $3(2 \%)$ & $6(4 \%)$ & 0.51 \\
\hline Admitted to hospital & $12(8 \%)$ & $30(20 \%)$ & $<0.01$ \\
\hline \multicolumn{4}{|l|}{ Causative organism of index UTI } \\
\hline Escherichia coli & $116(77 \%)$ & $112(74 \%)$ & 0.59 \\
\hline Klebsiella species & $18(12 \%)$ & $20(13 \%)$ & 0.72 \\
\hline Enterobacter species & $12(8 \%)$ & $14(9 \%)$ & 0.69 \\
\hline Citrobacter species & $3(2 \%)$ & $1(1 \%)$ & 0.63 \\
\hline Proteus species & $2(1 \%)$ & $4(3 \%)$ & 0.69 \\
\hline
\end{tabular}

${ }^{\mathrm{a}}$ Data are presented as numbers (percentages) except where noted

${ }^{\mathrm{b}}$ Hepatitis or cirrhosis

'COPD or chronic bronchitis

${ }^{\mathrm{d}}$ Receipt in the 6 months prior to index EB UTI presentation

Abbreviations: $B S I$ bloodstream infection, $C l$ confidence interval, ESC-R extended-spectrum cephalosporin-resistant, ESC-S extended-spectrum cephalosporinsusceptible, ED Emergency Department, IQR interquartile range, SNF skilled nursing facility, TMP-SMX trimethoprim-sulfamethoxazole, UTI urinary tract infection 


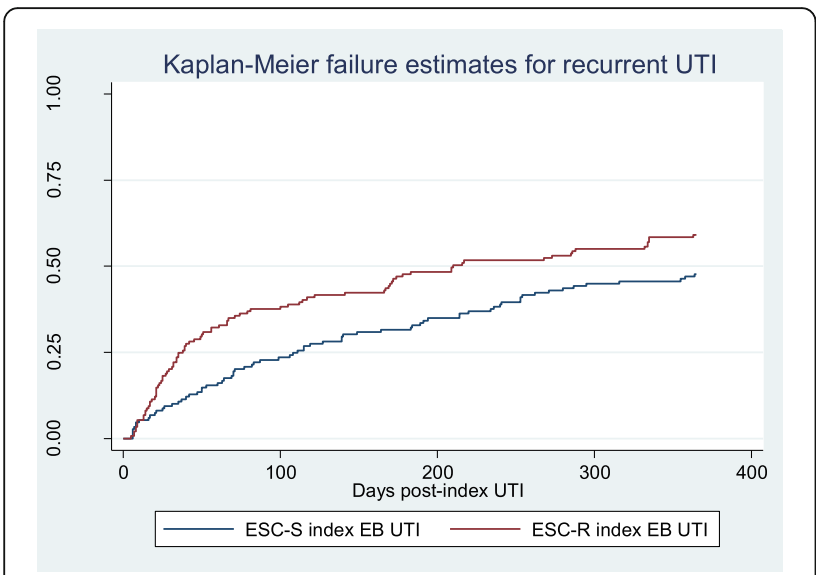

Fig. 1 Time to recurrent UTI stratified by exposure. Legend: Abbreviations: EB, Enterobacteriaceae; ESC-S, extended-spectrum cephalosporin-susceptible; ESC-R, extended-spectrum cephalosporinresistant; UTI, urinary tract infection

(Table 2), ESC-R EB UTI remained significantly associated with an increased hazard of recurrent UTI (adjusted HR [aHR] 1.39, 95\% CI 1.01-1.91, $P$ value $=0.04)$. Other factors that were associated with an increased hazard of recurrence included a history of UTI in the 6 months prior to the index UTI (aHR 1.59, 95\% CI 1.17$2.15, P$ value $<0.01)$ and a urinary catheter at the time of the index UTI (aHR 1.59, 95\% CI 1.06-2.38, $P$ value 0.03). There was a decreased hazard of recurrent UTI associated with a SNF stay in the 6 months prior to the index UTI (aHR 0.38, 95\% CI 0.19-0.77, $P$ value $=0.01$ ). Of note, presentation with pyelonephritis or bacteremia was not associated with a significantly increased hazard of recurrent UTI (pyelonephritis HR 0.96, 95\% CI 0.651.43; bacteremia HR 1.09, 95\% CI 0.45-2.68).

\section{Treatment received for the index UTI}

The most commonly prescribed antibiotics for the treatment of the index UTI were fluoroquinolones (100,33\%), nitrofurantoin $(94,31 \%)$, and trimethoprim-sulfamethoxazole (73, $24 \%)$. First-, third-, and fourth-generation cephalosporins were administered less frequently $(11,6,11 \%$ respectively).

Table 2 Multivariable Cox proportional hazards regression model of recurrent UTI

\begin{tabular}{lll}
\hline Variable & aHR $(95 \% \mathrm{Cl})$ & $P$ value \\
\hline ESC-R status & $1.39(1.01-1.91)$ & 0.04 \\
Prior UTI $^{\mathrm{a}}$ & $1.59(1.17-2.15)$ & $<0.01$ \\
Urinary catheter $^{\mathrm{b}}$ & $1.59(1.06-2.38)$ & 0.03 \\
Recent SNF stay $^{\mathrm{a}}$ & $0.38(0.19-0.77)$ & 0.01 \\
\hline
\end{tabular}

${ }^{\mathrm{a}}$ Within the 6 months prior to the index UTI

${ }^{b}$ Catheter in place at the time of the index UTI diagnosis

Abbreviations: aHR adjusted hazard ratio, ESC-R extended-spectrum

cephalosporin resistant, $\mathrm{Cl}$ confidence interval, SNF skilled nursing facility, UTI urinary tract infection
Of note, a similar proportion of the exposed and unexposed subjects received first- or third-generation cephalosporins: 17 (11\%) subjects received first-generation cephalosporins in both groups; 9 (6.0\%) of the exposed and $8(5.3 \%)$ of the unexposed subjects received a third-generation cephalosporin. More subjects in the exposed group received a fourth-generation cephalosporin (cefepime) than in the unexposed group (24 [17.7\%] exposed and 7 [5.1\%] unexposed).

Within the entire cohort, 158 patients (53\%) experienced IIAT. When IIAT was incorporated into the final multivariable Cox model for recurrent UTI (Table 3), we found that ESC-R status was no longer significantly associated with an increased hazard for recurrence (aHR $1.26,95 \%$ CI $0.91-1.76, P$ value $=0.17$ ). IIAT, however, was significantly associated with an increased hazard of recurrence (aHR 1.47, 95\% CI 1.04-2.06, $P$ value $=0.03$ ).

Due to the unexpected finding that prior SNF stay was associated with a decreased hazard of UTI recurrence, we also secondarily explored this relationship. We found that there were 12 patients in the cohort with a recent SNF stay, of which 11 were evaluated in the ED for their index UTI, 7 were admitted, and 7 received intravenous cefepime as the initial antibiotic. When treatment with cefepime was added to the multivariable model of UTI recurrence, prior SNF stay was no longer significantly associated with UTI recurrence (aHR for recent SNF stay $0.46,95 \%$ CI $0.18-1.18, P$ value $=0.11$.

\section{Discussion}

In this study, we found that patients who presented with a community-onset UTI due to an ESC-R organism had a significantly increased hazard of recurrent UTI within 12 months compared to those with an ESC-susceptible EB UTI. We also found that a greater proportion of patients with an ESC-R EB UTI experienced a delay in appropriate antibiotic initiation and that this impacted the relationship between ESC-R EB status and recurrence. More specifically, after accounting for IIAT, we found that the association between ESC-R status and recurrence was no longer significant, suggesting that the increased risk for recurrence with an ESC-R EB UTI may be related to the timing and selection of the treatment regimen.

To our knowledge, there are no prior studies that have specifically evaluated whether community-onset UTI with an ESC-R or ESBL-producing EB is associated with an increased risk for recurrence. There are prior studies demonstrating that with recurrent UTIs, there is increased risk for ultimately developing an ESC-R EB on urine culture $[5,11]$. Our study suggests that there may be a cyclic relationship, where the development of a UTI with an ESC-R EB organism increases the risk for further recurrence, related primarily to inappropriate treatment of such organisms and an associated lack of cure. 
Table 3 Multivariable Cox proportional hazards regression model of recurrent UTI including inappropriate initial antibiotic therapy

\begin{tabular}{lll}
\hline Variable & aHR $(95 \% \mathrm{Cl})$ & $P$ value \\
\hline ESC-R status & $1.26(0.91-1.76)$ & 0.17 \\
Prior UTI $^{\mathrm{a}}$ & $1.53(1.12-2.11)$ & 0.01 \\
Urinary catheter $^{\mathrm{b}}$ & $1.51(0.99-2.32)$ & 0.06 \\
Recent SNF stay $^{\mathrm{a}}$ & $0.36(0.17-0.75)$ & 0.01 \\
Inappropriate initial antibiotic therapy $^{\text {In }}$ & $1.47(1.04-2.06)$ & 0.03 \\
\hline
\end{tabular}

Within the 6 months prior to the index UTI

${ }^{b}$ Catheter in place at the time of the index UTI diagnosis

Abbreviations: $a H R$ adjusted hazard ratio, $C l$ confidence interval, ESC- $R$ extended-spectrum cephalosporin-resistance, SNF skilled nursing facility, UTI urinary tract infection

In addition to ESC-R status, we also found in our primary analysis that the hazard of recurrent UTI was significantly increased in the setting of prior UTIs (specifically a UTI diagnosed within 6 months of the index UTI). This finding is consistent with prior literature that has demonstrated that with each additional UTI, there is an incremental increase in the risk for another future UTI [8]. We also found that the presence of a urinary catheter was associated with an increased hazard of recurrence. This too is consistent with prior literature which has shown that urinary catheters are a significant risk factor for UTI as they create a portal of entry for bacteria into the genitourinary tract and may allow organisms to persist in the setting of biofilm production [10].

There are potential limitations of our study. Misclassification is a concern in retrospective studies. However, both the exposure and outcome were validated through medical record review by infectious diseases-trained physicians, rather than relying on diagnostic or billing codes. The assessment of the outcome was limited to review of the UPHS medical record. Patients who experienced clinical failure and sought care from outside providers would not have been captured. However, we found that $95 \%$ of the cohort had documented follow-up in our medical system. Further, the secondary analysis evaluating antibiotic use (IIAT) is potentially biased by confounding by indication. As a result, our analysis of IIAT was limited to secondary exploratory analyses for hypothesis-generation. Finally, because the present study was conducted in a single healthcare system, the results may not be generalizable to other dissimilar institutions.

\section{Conclusions}

The results of our study demonstrate that community-onset ESC-R EB UTIs are associated with an increased hazard of recurrent UTI compared to ESC-susceptible EB UTIs, even after adjusting for other factors that predispose to recurrence, and that this association may be related to the antibiotic regimen received for the index UTI. Further studies are needed to determine interventions that may reduce the risk of recurrence, including different antibiotic regimens and durations.

\section{Abbreviations \\ (aHR): Adjusted HR; (CDC): Centers for Disease Control and Prevention; \\ (CI): Confidence interval; (CLSI): Clinical and Laboratory Standards Institute; (EB): Enterobacteriaceae; (EDs): Emergency Departments; (ESBL): Extended- spectrum beta-lactamase; (ESC-R): Extended-spectrum cephalosporin resistance; (HR): Hazard ratio; (HUP): Hospital of the University of Pennsylvania; (IIAT): Inappropriate initial antibiotic therapy; (IQR): Interquartile range; (MDROs): Multidrug-resistant organisms; (MIC): Minimum inhibitory concentration; (NHSN): National Healthcare Safety Network; (PPMC): Penn Presbyterian Medical Center; (SNF): Skilled nursing facility; (UPHS): University of Pennsylvania Health System; (UTIs): Urinary tract infections}

\section{Acknowledgments}

Not applicable.

\section{Funding}

This work was supported by the Agency for Healthcare Research and Quality (grant number R18-HS020002 to E.L.); the National Institutes of Health (grant numbers K24-Al080942 to E.L., K01-Al103028 to J.H.H., K01-Al137317 to J.A.A.); the Centers for Disease Control and Prevention Cooperative Agreement Epicenters for the Prevention of Healthcare Associated Infections (grant number FOA\#CKO00163 to E.L.); and the Antibacterial Resistance Leadership Group (grant number 5 UM 1Al104681-05 with a subaward fellowship grant to J.A.A.). The funding bodies played no role in the design of the study, data collection, data analysis, interpretation of data, or writing of the manuscript.

\section{Availability of data and materials}

The datasets generated and/or analyzed during the current study are not publicly available in order to avoid loss of confidentiality for the included human subjects. The datasets may be available from the corresponding author on reasonable request and after review by an institutional review board.

\section{Authors' contributions}

JAA designed the study, performed data cleaning, data analysis, and wrote the manuscript. EL and JHH oversaw study design, analysis, and manuscript preparation. IN and CG performed susceptibility testing on the Enterobacteriaceae isolates. WB oversaw statistical analyses. JO, LD, and MW performed primary data collection and data cleaning. PT oversaw data collection and data cleaning. All authors read and approved the final manuscript.

\section{Ethics approval and consent to participate}

This study was approved by the University of Pennsylvania's Institutional Review Board (Protocol 812302). Verbal informed consent was obtained from study participants, rather than written consent, because consent was obtained at the time of a follow-up phone call after the patient's clinical visit for the UTI. Written informed consent was not feasible since the patients were no longer in the clinic/hospital when they were enrolled in the study.

\section{Consent for publication}

Not applicable.

\section{Competing interests}

The authors declare that they have no competing interests.

\section{Publisher's Note}

Springer Nature remains neutral with regard to jurisdictional claims in published maps and institutional affiliations.

\section{Author details}

'Division of Infectious Diseases, Department of Medicine; Center for Clinical Epidemiology and Biostatistics, Perelman School of Medicine, University of Pennsylvania, 719 Blockley Hall, 423 Guardian Drive, Philadelphia, PA 19104, USA. ${ }^{2}$ Division of Infectious Diseases, Department of Medicine; Center for Clinical Epidemiology and Biostatistics; Department of Biostatistics, 
Epidemiology and Informatics, Perelman School of Medicine, University of Pennsylvania, Philadelphia, PA, USA. ${ }^{3}$ Department of Pathology and Laboratory Medicine, Perelman School of Medicine, University of Pennsylvania, Philadelphia, PA, USA. ${ }^{4}$ Center for Clinical Epidemiology and Biostatistics; Department of Biostatistics, Epidemiology and Informatics, Perelman School of Medicine, University of Pennsylvania, Philadelphia, PA, USA. ${ }^{5}$ Center for Clinical Epidemiology and Biostatistics, Perelman School of Medicine, University of Pennsylvania, Philadelphia, PA, USA.

Received: 20 June 2018 Accepted: 11 February 2019

\section{Published online: 14 February 2019}

\section{References}

1. Simonsen L, Conn LA, Pinner RW, Teutsch S. Trends in infectious disease hospitalizations in the United States, 1980-1994. Arch Intern Med. 1998; 158(17):1923-8.

2. Talan DA, Krishnadasan A, Abrahamian FM, Stamm WE, Moran GJ, Group EMINS. Prevalence and risk factor analysis of trimethoprimsulfamethoxazole- and fluoroquinolone-resistant Escherichia coli infection among emergency department patients with pyelonephritis. Clin Infect Dis. 2008:47(9):1150-8.

3. Pitout JD, Nordmann P, Laupland KB, Poirel L. Emergence of Enterobacteriaceae producing extended-spectrum beta-lactamases (ESBLs) in the community. J Antimicrob Chemother. 2005;56(1):52-9.

4. Calbo E, Romani V, Xercavins M, Gomez L, Vidal CG, Quintana S, et al. Risk factors for community-onset urinary tract infections due to Escherichia coli harbouring extended-spectrum beta-lactamases. J Antimicrob Chemother. 2006:57(4):780-3.

5. Colodner R, Rock W, Chazan B, Keller N, Guy N, Sakran W, et al. Risk factors for the development of extended-spectrum beta-lactamase-producing bacteria in nonhospitalized patients. Eur J Clin Microbiol Infect Dis. 2004 23(3):163-7.

6. Apisarnthanarak A, Kiratisin P, Saifon P, Kitphati R, Dejsirilert S, Mundy LM. Clinical and molecular epidemiology of community-onset, extendedspectrum beta-lactamase-producing Escherichia coli infections in Thailand: a case-case-control study. Am J Infect Control. 2007;35(9):606-12.

7. Apisarnthanarak A, Kiratisin P, Mundy LM. Predictors of mortality from community-onset bloodstream infections due to extended-spectrum betalactamase-producing Escherichia coli and Klebsiella pneumoniae. Infect Control Hosp Epidemiol. 2008;29(7):671-4.

8. Beerepoot MA, ter Riet G, Nys S, van der Wal WM, de Borgie CA, de Reijke $T M$, et al. Lactobacilli vs antibiotics to prevent urinary tract infections: a randomized, double-blind, noninferiority trial in postmenopausal women. Arch Intern Med. 2012;172(9):704-12.

9. Drekonja DM, Rector TS, Cutting A, Johnson JR. Urinary tract infection in male veterans: treatment patterns and outcomes. JAMA Intern Med. 2013; 173(1):62-8

10. Foxman B. Urinary tract infection syndromes. occurrence, recurrence, bacteriology, risk factors, and disease burden Infect Dis Clin North Am. 2014; 28(1):1-13.

11. Rodriguez-Bano J, Alcala JC, Cisneros JM, Grill F, Oliver A, Horcajada JP, et al. Community infections caused by extended-spectrum beta-lactamaseproducing Escherichia coli. Arch Intern Med. 2008;168(17):1897-902.

12. $C D C / N H S N$. CDC/NHSN surveillance definitions for specific types of infections 2016. Available from: http://www.cdc.gov/nhsn/pdfs/pscmanual/ 17pscnosinfdef_current.pdf.

13. Performance standards for antimicrobial susceptibility testing M100-S20 CLSI. 2010.

Ready to submit your research? Choose BMC and benefit from:
- fast, convenient online submission
- thorough peer review by experienced researchers in your field
- rapid publication on acceptance
- support for research data, including large and complex data types
- gold Open Access which fosters wider collaboration and increased citations
- maximum visibility for your research: over 100M website views per year
At BMC, research is always in progress.
Learn more biomedcentral.com/submissions

